\title{
Intelligent Chatting Mobile Robot for seniors and Kids \\ Ying $\mathrm{Xu}$ \\ North China Electric Power University, Beijing, 102206 \\ alanncepu@foxmail.com
}

Keywords: Robot; Intelligent voice chat; Wheeled movement; The elderly and children

\begin{abstract}
With the expansion of robot applications, the task done by the robot is more complicated. In order to meet the needs of special groups such as the elderly and children, we combine the mobile voice software development kit (SDK) of Xunfei platform with Google's software development Tool to create a voice interaction APP which can implement voice dictation, speech recognition, speech synthesis and speech resolution. At the same time, connect the Android Bluetooth and the HC305 Bluetooth connected to single-chip serial port for analog serial communication. Then Arduino converts the voice text to a four-digit voltage signal that controls the L298N drive module to drive the DC motor motion. Therefore, the robot can perform intelligent voice chat and wheeled movement simultaneously.
\end{abstract}

\section{Technical Implementation of Wheeled Movement}

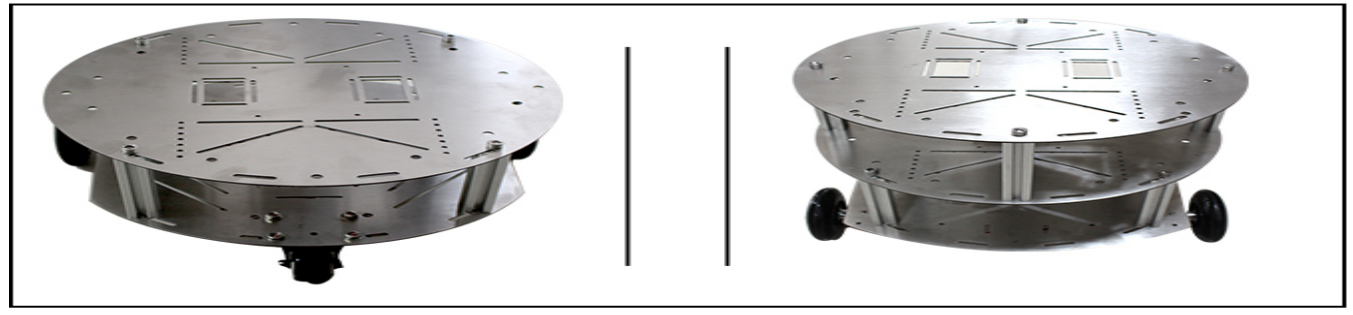

Figure1.1 Robot development platform

With a multi-layer stack mechanical structure, the robot that takes PC104 embedded computer as the core control system is driven by two rounds of differential drive. The servo control system mainly includes PWM power drive, velocity measurement unit, power supply system and sensor information acquisition module, etc. We can combine the existing wheeled robot chassis development with Arduino electronic platform, so that the robot completes the wheeled movement. Here, we use the remote control device as the output device of Arduino. Arduino, in conjunction with its own source program, performs an uninterrupted scan of each cycle signal so that the remote control controls the movement of the robot at each point in time. On this basis, we will also install the photocell on both sides of the wheel. Then, we can use the difference between the speeds of the wheel caused by the difference in voltage to control the robot turning a specific angle.

\section{The Movement Model of the Robot}

Before implementing control with code, we'd better start with a simple understanding of the principle involved. To be exact, this 4WD wheeled robot uses the so-called "Skid-steer Drive" that is a type of movement similar to the Differential Drive.

Referring to Ackerman's geometrical principle, four tires are approximately revolved around a central point when the car is turned to ensure the stability of the vehicle. But it's hard to guarantee that because the four-wheel differential steering car has no steering mechanism and it is very easy to slip when the car turns. Considering the car's centroid as a center of mass and ignoring the impact of changes in the road, we can draw four rounds of differential kinetic models of the car as shown below. 


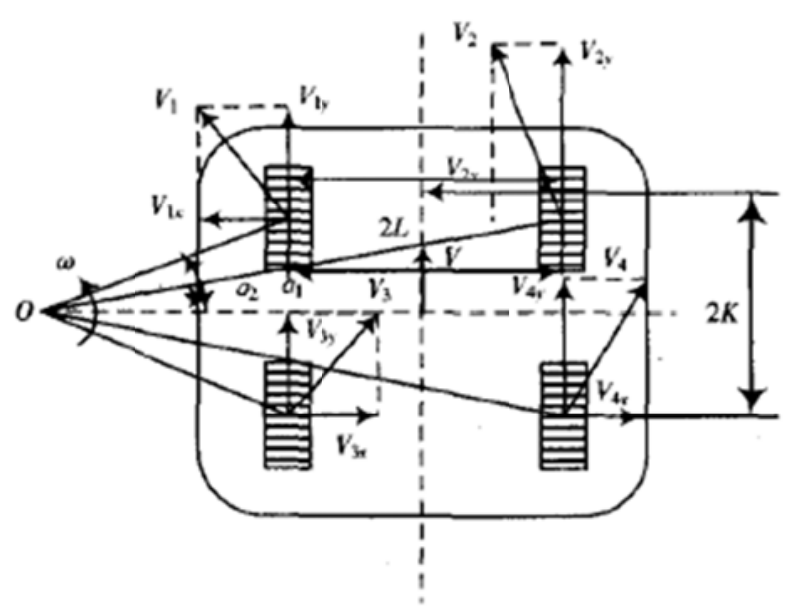

Figure1.2 Robot kinematics model

In the figure, $\alpha_{1}$ is the angle of the front left wheel and the rear left wheel; $\alpha_{2}$ is the angle of the front right wheel and the rear right wheel; $2 \mathrm{~L}$ is the distance between the left and right wheels; $2 \mathrm{~K}$ is the wheelbase between front and rear wheels; $\mathrm{V}$ is the linear velocity of the center of mass of the car; $\mathrm{W}$ is the angular velocity of the center of mass of the car; $V_{1}, V_{2}, V_{3}, V_{4}$ is the actual motion direction of each wheel center; According to the figure above, the relationship between velocity and rotation angle can be obtained as follows:

$$
\begin{aligned}
& V_{1}=\mathrm{W} \cdot R_{1}=\mathrm{W} \cdot \mathrm{K} \sin \alpha_{1} \\
& V_{2}=\mathrm{W} \cdot R_{1}=\mathrm{W} \cdot \mathrm{K} \sin \alpha_{2} \\
& V_{3}=V_{1}=W \cdot K \sin \alpha_{1} \\
& V_{4}=V_{2}=W \cdot K \sin \alpha_{2}
\end{aligned}
$$

From the formula $V_{\mathrm{y}}=V \cdot \cos \alpha$,we know:

$$
\begin{aligned}
& V_{1 y}=V_{1} \cdot \cos \alpha_{1}=W \cdot K \tan \alpha_{1}=W(R-L) \\
& V_{2 y}=V_{2} \cdot \cos \alpha_{2}=W \cdot K \tan \alpha_{2}=W(R+L) \\
& V_{3 y}=V_{2} \cdot \cos \alpha_{1}=W \cdot K \tan \alpha_{1}=W(R-L) \\
& V_{4 y}=V_{4} \cdot \cos \alpha_{2}=W \cdot K \operatorname{Kan} \alpha_{2}=W(R+L)
\end{aligned}
$$

Among them, $\mathrm{R}=\mathrm{VW}$.

Then, the angular velocity of the motor is :

$$
W_{n}=V_{n y} \bullet i \bullet r, n=1,2,3,4
$$

Among them, $i$ is the reduction ratio of the motor; $r$ is the radius of the wheel; The speed of the motor can be obtained from $\mathrm{n}=\mathrm{W} \cdot 2 \pi$.

Calculation of Wheel Speed. After understanding the principle of steering, we can calculate the date we want according to the size of the actual car. The parameters of the car I used are as follows:

The wheelbase between the front and rear wheels $2 \mathrm{~K}=168 \mathrm{~mm}$

The distance between the left and right wheels $2 \mathrm{~L}=266 \mathrm{~mm}$

The wheel diameter $\mathrm{r}=130 \mathrm{~mm}$

Motor reduction ratio $i=1: 30$

Here, we specify the angular velocity $\mathrm{W}=5 \mathrm{rad} / \mathrm{s}$ and the turning radius $\mathrm{r}=100 \mathrm{~mm}$ when the car turns. From the above formula we can obtain the speed of each wheel:

$$
n_{1}=n_{3}=18.3 \mathrm{~m} / \mathrm{s} ; n_{2}=n_{4}=116.1 \mathrm{~m} / \mathrm{s}
$$

When the right-turn message is received, the inner wheel speed is set to 18 and the outer wheel speed is set to 116.The left-turn is similar. 
Hardware Connection. Connect the interface of the motor drive board L293D and the corresponding interface of the Arduino board. The specific pin connection is as follows:

$\Delta$ If you only want to use DC / stepper motor, you should connect the following pins:

Digital port 11:Dc motor\#1/Step by step\#1 (PWM)

Digital port 3 :Dc motor\#2/Step by step\#1 (PWM)

Digital port $5:$ Dc motor\#3/Step by step\#2 (PWM)

Digital port 6 :Dc motor\#4/Step by step\#2 (PWM)

$\Delta$ If you also want to control the DC / stepper motor, the following pins should be added:

Digital port 4: DIR CLK trigger

Digital port 7: The allowed port of the DIR EN directive

Digital port 8: DIR SER

Digital port 12: DIR ATCH interrupt connection

In addition, GND and 5V pins must also be connected, otherwise we cannot control the DC motor stably.

Code for Testing. First compile and upload the code in Arduino, and then run the following command to specify the serial port that the Arduino board connects to.

Start the node manager (rescore) before running the node.

Run: rosrun rosserial_python serial_node.py/dev/ttyACM0.

The last parameter depends on the serial port selected in the Arduino IDE, where we use ACM0. Then run: rosrun turtlesim turtle_teleop_key, Using the arrow keys, you can control the robot freely. Sensor Information Acquisition Module. Infrared photoelectric sensor (referred to as photoelectric sensor, also known as photoelectric switch) achieves control by converting light intensity changes into electrical signal changes. Photoelectric sensors are typically made up of three parts, which are divided into: transmitters, receivers, and detection circuits.

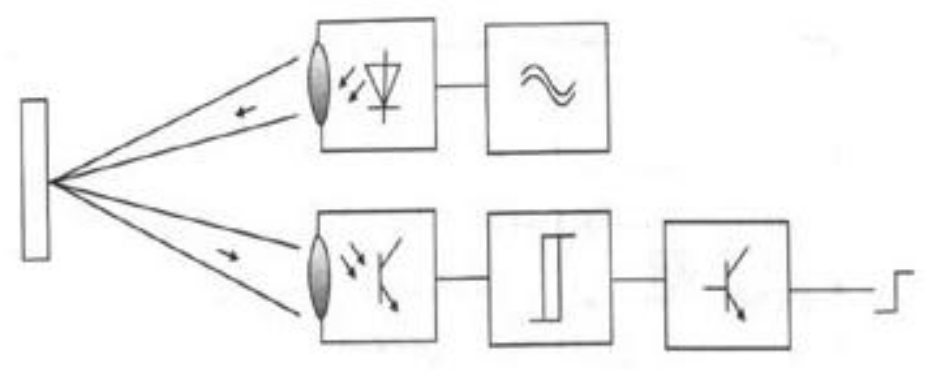

Figure1.3 Infrared photoelectric sensor

The emitter emits infrared light to the receiver, forming a row of light curtains. When the light curtain is blocked, the receiver will detect a certain light intensity changes and determine that there are foreign objects into the dangerous area set by the infrared sensor. Then, it immediately outputs a control signal, which is transmitted to the production equipment controller via cable, and stops the device from operation or prevents false start.

Photoelectric switch (photoelectric sensor) is the abbreviation of photoelectric proximity switch. It uses the detected object to block or reflect the light beam, by the synchronous loop gating circuit, thus detecting the presence of objects. Objects are not limited to metal, and all objects that reflect light can be detected. The photoelectric switch converts the input current into an optical signal on the transmitter, and the receiver detects the target object based on the presence or absence of the received light.

\section{Technical Implementation of Voice Interaction}

With reference to the open voice semantic system on Xunfei platform and online word synthesis system, we have developed a voice interactive APP which enters a knowledge language library for the robot. In the interactive process, the language is first converted to text by the voice recognition 
service. After that, the semantic recognition service analyzes its grammar, and obtains a syntactic structure of "subject + predicate + object + adverb". The robot follows the algorithm and combines the information in the knowledge library to get the answers that the user wants, and thus completes the communication with the human. In the interactive process, in order to make users more pleasant, you can also introduce some interesting answers.

\section{References}

[1] Chen Wei, Jiang Xuyun. Simulation of Wheel Robot Path Planning Control [J]. Computer Simulation, 2016, 33(5):367-371.

[2] Jin Zuguang, Chen Chao, Tang Jian. Design of RFID - Voice Interactive System for Indoor Blind [J]. Automated Instrumentation, 2014, 35(3):73-76.

[3] Hu Juntao, Li Yarong, Li Lin. Research on Trajectory Tracking Control of Wheeled Mobile [J]. Instrumentation Technology, 2013(4):27-29.

[4] Zong Zhaohui, Zhang Haihong, Li Zhibin. Research on Omni - Directional Mobile Mechanism of Wheeled Robot [J].Machine Manufacturing, 2011, 49(3):14-16.

[5] Hamza KHAN, Jamshed IQBAL, Khelifa BAIZID,Etc. Longitudinal and Lateral Slip Control of Autonomous Wheeled Mobile Robot Path Tracking (English)[J]. Journal of Zhejiang University-Science C (Computers \& Electronics), 2015(2).

[6] Shi Xiuen. Research on Motion Control and Location Method of Wheeled Mobile Robot [D]. Xi'an University of Technology, 2006.

[7] Bao Yanyan. Research and Design of Robot Voice Interactive System [D]. Northeastern University, 2012.

[8] Li Xiang, Li Xin, Hu Chen,Etc. Design and Implementation of Teager Speech and Emotional Interactive System for Intelligent Robot[J]. Journal of Instrument \& Instrumentation, 2013, 34(8):1826-1833.

[9] Yuan Jian, He Xiang, Xu Huahu,Etc. Research on Speech and Emotional Recognition and Interaction Technology of Service[J]. Small Microcomputer System, 2010, 31(7).

[10] Lu Tianzeng. Research on Interactive Technology of Intelligent Robot Based on Android [D]. Ocean University of China, 2015. 